\title{
Erratum to: "In vitro Antiviral Activity of Recombinant Antibodies of IgG and IgA Isotypes to Hemagglutinin of the Influenza A Virus" [Molecular Biology 51, 804 (2017)]
}

\author{
V. V. Argentova ${ }^{a, *}$, T. K. Aliev ${ }^{b}$, V. V. Zarubaev ${ }^{c}$, S. A. Klotchenko ${ }^{c}$, A. A. Shtro ${ }^{c}$ M. V. Sergeeva ${ }^{c}$, \\ V. A. Toporova ${ }^{d}$, D. A. Dolgikh ${ }^{a, d}$, P. G. Sveshnikov ${ }^{e}$, A. V. Vasin ${ }^{c}$, and M. P. Kirpichnikov ${ }^{a, d}$ \\ ${ }^{a}$ Department of Bioengineering, Faculty of Biology, Moscow State University, Moscow, 119234 Russia \\ ${ }^{b}$ Department of Chemical Enzymology, Faculty of Chemistry, Moscow State University, Moscow, 119991 Russia \\ ${ }^{c}$ Research Institute of Influenza, Ministry of Healthcare of the Russian Federation, St. Petersburg, 197376 Russia \\ ${ }^{d}$ Shemyakin-Ovchinnikov Institute of Bioorganic Chemistry, Russian Academy of Sciences, Moscow, 117997 Russia \\ ${ }^{e}$ Russian Research Center for Molecular Diagnostics and Therapy, Moscow, 117149 Russia \\ *e-mail: vicarg@rambler.ru \\ Submitted July 25, 2018; accepted for publication July 26, 2018
}

DOI: $10.1134 / \mathrm{S} 0026893318090017$

The name of the penultimate author should read $A$. V. Vasin.

The original article can be found online at

$10.1134 / \mathrm{S} 0026893317060024$ 